\title{
Static deformation of an orthotropic elastic layered medium due to a non- uniform discontinuity along a very long strike-slip fault
}

\author{
Shamta Chugh ${ }^{1,}$ Dinesh Kumar Madan ${ }^{2, *}$ and Kuldip Singh ${ }^{1}$ \\ ${ }^{1}$ Department of Mathematics, Guru Jambheshwar University of Science and Technology, Hisar-125001, INDIA. \\ ${ }^{2}$ Department of Mathematics, The Technological Institute of Textile and Sciences, Bhiwani-127021, INDIA. \\ "Corresponding Author email: dk_madaan@rediffmail.com,dineshmadan@titsbhiwani.ac.in
}

\begin{abstract}
Closed-form analytic expressions for the deformation field at any point of a homogeneous, orthotropic, homogeneous elastic layer interfacing differently to a base due to non-uniform discontinuity (slip) along a very long strike-slip fault situated in the orthotropic elastic layer have been obtained. Four non-uniform slip profiles: parabolic, linear, cubic and elliptic along the fault are considered. Numerically, for each slip profiles the results for the present model (welded contact) are compared with the corresponding results for uniform orthotropic elastic half-space. Contour maps for different slip profiles for each type of interfacing are presented.
\end{abstract}

Keywords: orthotropic medium; strike-slip; non-uniform slip; half-space; contour.

\section{Introduction}

Two-dimensional dislocation models of faulting are useful to explain the observed co-seismic deformations. Strike-slip faults are faults with very little vertical component of motion, i.e.: the slip vector is nearly parallel to the strike direction. Strike-slip faults are typically steep or vertical and in Andersonian fault theory are associated with a stress regime where both maximum and minimum stresses are near horizontal. Many strike slip faults are idealized as being vertical cuts going, presumably, all the way to the core-mantle boundary. In truth, the geometry of apparently vertical strike slip faults is quite variable with depth. Elastic deformability combined with sample asymmetry, i.e., the mechanical environment of all points on the surface are not identical, can cause non-uniform slip when there is a uniform change in shear stress. Even if a sample has perfect symmetry, i.e., all points on the slip surface are identical to all others in all properties and loading, uniform slip is not ensured. Uniform slip could be unstable to small spatial perturbations, thus leading to spatially non-uniform slip (Ruina et al, 1986).

The static deformation of a semi-infinite elastic isotropic elastic medium due to a very long strike-slip and dip-slip fault has been studied by many researchers (Kasahara, 1964 and others). However, most of these studies assumed uniform slip on a fault. The assumption of uniform slip makes the edges of the fault plane discontinuous where the displacement is indeterminate and the stress is infinite. For this reason, uniform slip models cannot be used in the vicinity of the fault (near field). There are a number of interesting phenomenon that occur near the edge of the fault zone, e.g., vertical movements associated with strike-slip faulting. In order to study these phenomena, it is necessary to consider models of earthquake faulting with non-uniform slip on a fault. In the variable slip models the amount of slip in a given place and the length of rupture may vary from earthquake to earthquake.

Evidence for non-uniform slip histories of faults and in particular for periods of pronounced earthquakes clustering on different time scales are provided by paleoseismologic studies for different type of faults such as thrust faults, normal faults and strike-slip faults. The Nankaido earthquake (1707) demonstrates that the slip in a given place may vary significantly between earthquakes, suggesting the variable slip model. Starr (1928) proposed a two-dimensional elliptical crack model in an infinite medium. Yang and Toks $\ddot{O}$ z (1981) used finite-element method to study the trapezoidal type nonuniform slip on a strike-slip fault in an isotropic elastic half-space. Wang and Wu (1983) obtained closed-form analytical solution for displacement and stress fields due to a non-uniform slip along a strike-slip fault for the same model. Singh et al (1994) obtained closed-form analytical expressions for displacements due to non-uniform slip on a long vertical strike-slip and dip-slip 
faults in a uniform isotropic elastic half-space. Madan et al (2005) obtained static deformation field due to non-uniform slip (parabolic, linear, cubic and elliptic) on a long vertical strike-slip fault in an orthotropic elastic half-space.

In the present paper, the static deformation field caused by non-uniform slip profiles (parabolic, linear, cubic and elliptic) along a very long vertical strike-slip fault situated in a homogeneous, orthotropic, linear elastic layer which is in perfect(welded) contact with homogeneous, orthotropic, elastic half-space has been obtained. The results for 'smooth-rigid' or 'rough-rigid' contact have also been obtained as a particular case. Another particular case is also considered when the layer and half-space become identical, i.e. uniform orthotropic elastic half-space.. The variation of the displacements with the horizontal distance from the fault embedded in the layer due to different slip profiles has been compared numerically with the displacements due to fault situated in a uniform orthotropic elastic half-space. Contour maps showing the displacement field around the vertical strike-slip fault lying in an orthotropic elastic layer for different slip profiles in case of 'perfect', 'smooth-rigid' and 'rough-rigid' interfacing are presented. The results for an isotropic case can be derived as a particular case from these results. Permanent surface deformations which occur as a result of faulting can be measured from geodetic surveys carried out before and after an earthquake. Using inversion, these expressions can be used to obtain the fault parameters such as slip, width and depth etc.

\section{Formulation and solution of the problem}

Consider a semi-infinite medium consisting of a homogenous orthotropic elastic horizontal layer of thickness $H$ lying over a homogenous orthotropic elastic half-space. The origin of the Cartesian co-ordinate system $(x, y, z)$ is placed at the upper boundary of the semi-infinite medium with the $Z$-axis vertically downwards (Figure 1 ). The layer, therefore, occupies the region $0 \leq z<H$ and the half-space $z>H$. It is assumed that an orthotropic elastic horizontal layer of uniform thickness $H$ is coupling in different ways such as 'perfectly bonded'(welded) or 'smooth-rigid' or 'rough-rigid' to the base. Assuming that a long vertical strike-slip fault of infinite length with strike along the $x$-axis and of finite width (depth) occupying the region $-\infty<x<\infty, y=0$ and $0 \leq z \leq d$ lies completely in the orthotropic horizontal elastic layer. Let the slip (dislocation) on the fault be denoted by $b$, which is not uniform in general. We are considering a 2-D approximation in which $\mathrm{b}$ is independent of $x$.

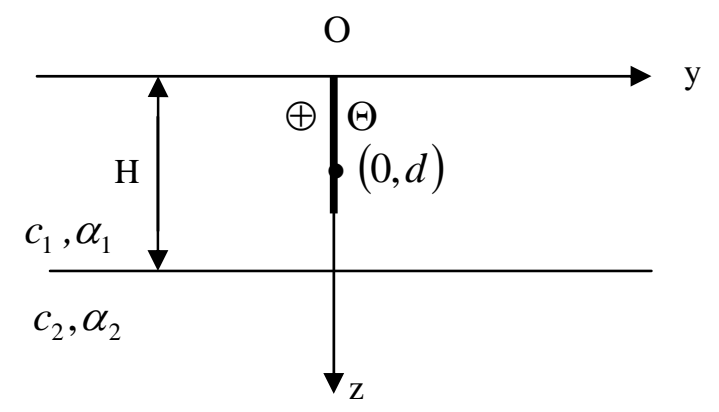

Fig.1. Geometry of a long vertical strike-slip fault of finite depth 'd' in a homogenous orthotropic elastic layer ( $0 \leq z<H$ ) of uniform thickness $H$ lying over a homogenous orthotropic elastic half-space ( $z>H$ ).

$\oplus$ and $\Theta$ indicate the displacements in the positive x-direction and negative x-direction respectively.

The boundary surface $Z=0$ is horizontal plane and is a plane of elastic symmetry. It is further assumed that the bounding plane $Z=0$ is traction free, so that

$$
\tau_{13}=0 \text { at } \mathrm{z}=0
$$

and for each type of interfacing, the conditions at $z=H$ are:

\subsection{Perfect contact}

When the interface between the orthotropic elastic layer and the half-space at $z=H$ is 'perfect', the displacement $u$ and stress $\tau_{13}$ across the horizontal plane $z=H$ are continuous. That is,

$$
\begin{aligned}
& u\left(z=H^{+}\right)=u\left(z=H^{-}\right), \\
& \tau_{13}\left(z=H^{+}\right)=\tau_{13}\left(z=H^{-}\right) .
\end{aligned}
$$




\subsection{Smooth-rigid Contact}

When the orthotropic elastic layer is in 'smooth' contact with a lower semi-infinite rigid medium at the interface $z=H$, the condition is

$$
\tau_{13}=0 \text { at } \mathrm{z}=H .
$$

\subsection{Rough-rigid Contact}

When the orthotropic elastic layer is in 'rough' contact with a lower semi-infinite rigid base at the interface $Z=H$, the condition is

$$
u=0 \text { at } \mathrm{z}=H .
$$

For antiplane strain deformation, the displacement u parallel to $x$-axis, at any point $(y, z)$ of an orthotropic elastic infinite medium, due to concentrated line force $\mathrm{F}$, acting in the $x$-direction, passing through the point $\left(\xi_{2}, \xi_{3}\right)$ is given by (Garg et al,1996)

$$
u^{(1)}=\frac{F}{2 \pi \alpha c} \int_{0}^{\infty}\left(\frac{1}{k}\right) e^{-k \alpha\left|z-\xi_{3}\right|} \cos k\left(y-\xi_{2}\right) d k
$$

where

$$
c_{55}=c, c_{66}=c \alpha^{2} \text {. }
$$

$\mathrm{C}_{55}$ and $\mathrm{C}_{66}$ are rigidities of an orthotropic elastic medium and (1) denotes the displacement field in the $\mathrm{x}$-direction.

By force-dislocation equivalence theorem the displacement field generated by shear dislocation on an infinitesimal surface element is the same as that generated by a couple applied at the surface element in the absence of dislocation (Eshelby,1957 and Burridge \& Knopoff , 1964).

At the point $\left(\xi_{2}, \xi_{3}\right)$, there acts a two-dimensional line source- either a single couple (12) or a single couple (13). The displacements $u^{(12)}$ and $u^{(13)}$, parallel to the x-axis, due to the single couple (12) of moment $F_{12}$ and due to the single couple (13) of moment $F_{13}$, respectively, are found to be

$$
\begin{aligned}
& u^{(12)}=\frac{F_{12}}{2 \pi \alpha c} \int_{0}^{\infty} e^{-k \alpha\left|z-\xi_{3}\right|} \sin k\left(y-\xi_{2}\right) d k, \\
& u^{(13)}=\frac{ \pm F_{13}}{2 \pi \alpha c} \int_{0}^{\infty} e^{-k \alpha\left|z-\xi_{3}\right|} \cos k\left(y-\xi_{2}\right) d k .
\end{aligned}
$$

These displacements (parallel to the $X$-axis and due to the line source of single couple (12) or (13)) can be unified into the following integral

$$
u_{0}=\int_{0}^{\infty}\left[A_{0} \sin k\left(y-\xi_{2}\right)+B_{0} \cos k\left(y-\xi_{2}\right)\right] e^{-\alpha k\left|z-\xi_{3}\right|} d k
$$

The source coefficients $A_{0}$ and $B_{0}$ for various two-dimensional buried sources are given in the following Table 1.:

Table 1. Source Coefficients for Various Seismic Sources

\begin{tabular}{llc}
\hline Source & $A_{0}$ & $B_{0}$ \\
\hline Single couple (12) & $\frac{F_{12}}{2 \pi \alpha c}$ & 0 \\
Single couple (13) & 0 & $\frac{ \pm F_{13}}{2 \pi c}$ \\
\hline
\end{tabular}


For a line dislocation source acting at the point $\left(\xi_{2}, \xi_{3}\right)$ of the layer, suitable expressions for the horizontal displacements $u$ in the layer and the half-space are (Garg and Sharma, 1992)

$$
\begin{aligned}
u^{I}=u_{0} & +\int_{0}^{\infty}\left[A_{1} \sin k\left(y-\xi_{2}\right)+B_{1} \cos k\left(y-\xi_{2}\right)\right] e^{-\alpha_{1} k z} d k \\
& +\int_{0}^{\infty}\left[C_{1} \sin k\left(y-\xi_{2}\right)+C_{1} \cos k\left(y-\xi_{2}\right)\right] e^{\alpha_{1} k z} d k
\end{aligned}
$$

for $0 \leq z<H$ and for $z>H$

$$
u^{I I}=\int_{0}^{\infty}\left[A_{2} \sin k\left(y-\xi_{2}\right)+B_{2} \cos k\left(y-\xi_{2}\right)\right] e^{-\alpha_{2} k z} d k
$$

The superscript I denotes for the layer and superscript II denotes for the half-space. The coefficients $A_{1}$, $B_{1}$ etc. for each type of interfacing can be determined by using the respective conditions given above.

\subsection{A very long vertical Strike-Slip Fault}

The displacement field due to a very long strike-slip fault of arbitrary orientation is given by (Maruyama, 1966)

$$
u=\int_{L} \Delta u G_{1 k}^{1} n_{k} d s
$$

where the summation convention has been used $(\mathrm{k}=2,3)$ only. $\Delta \mathrm{u}$ is the displacement discontinuity parallel to the strike-slip fault in the $\mathrm{x}$-direction. $\mathrm{n}_{\mathrm{k}}$ is the unit normal to fault section $\mathrm{L}, d s$ is the width of line fault and the Green function $\mathrm{G}_{1 \mathrm{k}}^{1}$ are given by (Garg et al,1996)

$$
\mathrm{G}_{12}^{1}=\mathrm{c}_{66} \frac{\partial \mathrm{u}^{(1)}}{\partial \xi_{2}}, \mathrm{G}_{13}^{1}=\mathrm{c}_{55} \frac{\partial \mathrm{u}^{(1)}}{\partial \xi_{3}}
$$

in which $u^{(1)}$ [given by equation (5)] is the displacement due to a concentrated line-force of unit magnitude, per unit length, acting in the x-direction.

Writing $\Delta u=b, n_{2}=-\sin \delta$ and $\mathrm{n}_{2}=\cos \delta$, where $\delta$ is the dip of the fault. Then equation (9) becomes

$$
u=\int_{L} b\left(G_{13}^{1} \cos \delta-G_{12}^{1} \sin \delta\right) d s
$$

Thus the Green's function $G_{12}^{1}$ corresponds to a vertical strike-slip fault and, represents upto a dimensional constant, the xcomponent of the displacement at the point $(\mathrm{y}, \mathrm{z})$ due to a single couple (12) at the point $\left(\xi_{2}, \xi_{3}\right)$ and similarly, $\mathrm{G}_{13}^{1}$ corresponds to a horizontal strike-slip fault and, represents upto a dimensional constant, the x-component of the displacement at the point $(\mathrm{y}, \mathrm{z})$ due to a single couple (13) at the point $\left(\xi_{2}, \xi_{3}\right)$.

Using interface conditions (1)-(2) in equations (10)-(11) and from equations (5), (13) and (14), we obtain the following expressions of displacements for perfect contact due to very long vertical strike-slip fault situated in the layer at a point $(0, h)$ as: 


$$
\begin{aligned}
u^{I}=-\frac{\alpha_{1}}{2 \pi} \int_{0}^{d} b(h) & {\left[\sum_{n=0}^{\infty} T^{n}\left(\frac{y}{y^{2}+\left[\alpha_{1}(2 n H+z-h)\right]^{2}}+\frac{y}{y^{2}+\left[\alpha_{1}(2 n H+z+h)\right]^{2}}\right)\right.} \\
& \left.+\sum_{n=1}^{\infty} T^{n}\left(\frac{y}{y^{2}+\left[\alpha_{1}(2 n H-z-h)\right]^{2}}+\frac{y}{y^{2}+\left[\alpha_{1}(2 n H-z+h)\right]^{2}}\right)\right] d h
\end{aligned}
$$

for $0 \leq z<H$ and for $z>H$

$$
\begin{aligned}
u^{I I}=\frac{-\alpha_{1}}{2 \pi} \int_{0}^{d} b(h)\left[\sum _ { n = 0 } ^ { \infty } ( 1 + T ) \Gamma ^ { n } \left(\frac{y}{y^{2}+\left[\alpha_{1}(2 n H+H+h)+\alpha_{2}(z-H)\right]^{2}}\right.\right. \\
\left.\left.+\frac{y}{y^{2}+\left[\alpha_{1}(2 n H+H-h)+\alpha_{2}(z-H)\right]^{2}}\right)\right] d h
\end{aligned}
$$

where

$$
T=\frac{c_{1} \alpha_{1}-c_{2} \alpha_{2}}{c_{1} \alpha_{1}+c_{2} \alpha_{2}}
$$

Let the various slip profiles along the fault (discontinuities in the displacements on the crack) are varying accordingly as:

$$
\begin{array}{ll}
\text { Uniform slip: } & b(h)=b_{0}, \text { (constant) } \\
\text { Parabolic slip: } & b(h)=b_{0}\left(1-h^{2} / d^{2}\right), \\
\text { Linear slip: } & b(h)=b_{0}(1-h / d), \\
\text { Elliptic slip: } & b(h)=b_{0}\left(1-h^{2} / d^{2}\right)^{1 / 2}, \\
\text { Cubic slip: } & b(h)=b_{0}\left(1-h^{2} / d^{2}\right)^{3 / 2}
\end{array}
$$

where $0<h<d$.

By substituting the value of $b(h)$ for different slip profiles in the equations (15) and (16) and then integrating with the help of Wolfram Mathematica and Standard transform integrals over the limit $0<h<d$, we obtain the following closed-form expressions for the displacements at any point $(y, z)$.

\subsubsection{Uniform slip}

$$
\begin{aligned}
u^{I}=\frac{b_{0}}{2 \pi} & {\left[\sum_{n=0}^{\infty} T^{n}\left\{\tan ^{-1} \frac{(2 n H+z-d) \alpha_{1}}{y}-\tan ^{-1} \frac{(2 n H+z+d) \alpha_{1}}{y}\right\}\right.} \\
+ & \left.\sum_{n=1}^{\infty} T^{n}\left\{\tan ^{-1} \frac{(2 n H-z-d) \alpha_{1}}{y}-\tan ^{-1} \frac{(2 n H-z+d) \alpha_{1}}{y}\right\}\right],
\end{aligned}
$$

for $0 \leq Z<H$ and for $Z>H$

$$
\begin{aligned}
u^{I I}=\frac{b_{0}}{2 \pi}\left[\sum _ { n = 0 } ^ { \infty } ( 1 + T ) T ^ { n } \left\{\tan ^{-1}\right.\right. & \frac{\alpha_{1}(2 n H+H-d)+\alpha_{2}(z-H)}{y} \\
& \left.\left.-\tan ^{-1} \frac{\alpha_{1}(2 n H+H+d)+\alpha_{2}(z-H)}{y}\right\}\right]
\end{aligned}
$$


2.4.2 Parabolic slip

$$
\begin{aligned}
u^{I}=\frac{b_{0}}{2 \pi \alpha_{1}}\left[\sum _ { n = 0 } ^ { \infty } T ^ { n } \left\{\alpha _ { 1 } \left(1+\frac{y^{2}}{\alpha_{1}^{2} d^{2}}\right.\right.\right. & \left.-\frac{(2 n H+z)^{2}}{d^{2}}\right) \\
& \times\left(\tan ^{-1} \frac{(2 n H+z-d) \alpha_{1}}{y}-\tan ^{-1} \frac{(2 n H+z+d) \alpha_{1}}{y}\right) \\
& \left.-\frac{y(2 n H+z)}{d^{2}} \log \left(\frac{y^{2}+(2 n H+z+d)^{2} \alpha_{1}^{2}}{y^{2}+(2 n H+z-d)^{2} \alpha_{1}^{2}}\right)+\frac{2 y}{d}\right\} \\
+\sum_{n=1}^{\infty} T^{n}\left\{\alpha_{1}\left(1+\frac{y^{2}}{\alpha_{1}^{2} d^{2}}-\frac{(2 n H-z)^{2}}{d^{2}}\right)\right. & \times\left(\tan ^{-1} \frac{(2 n H-z-d) \alpha_{1}}{y}-\tan ^{-1} \frac{(2 n H-z+d) \alpha_{1}}{y}\right) \\
& \left.\left.-\frac{y(2 n H-z)}{d^{2}} \log \left(\frac{y^{2}+(2 n H-z+d)^{2} \alpha_{1}^{2}}{y^{2}+(2 n H-z-d)^{2} \alpha_{1}^{2}}\right)+\frac{2 y}{d}\right\}\right]
\end{aligned}
$$

for $0 \leq Z<H$ and for $Z>H$

$$
\begin{aligned}
u^{I I}= & \frac{b_{0}}{2 \pi \alpha_{1}}\left[\sum _ { n = 0 } ^ { \infty } ( 1 + T ) T ^ { n } \left\{\alpha_{1}\left(1+\frac{y^{2}}{\alpha_{1}^{2} d^{2}}-\frac{\left[\alpha_{1}(2 n H+H)+\alpha_{2}(z-H)\right]^{2}}{\alpha_{1}^{2} d^{2}}\right)\right.\right. \\
& \times\left\{\tan ^{-1} \frac{\alpha_{1}(2 n H+H-d)+\alpha_{2}(z-H)}{y}-\tan ^{-1} \frac{\alpha_{1}(2 n H+H+d)+\alpha_{2}(z-H)}{y}\right\} \\
& \left.\left.-\frac{y\left\{\alpha_{1}(2 n H+H)+\alpha_{2}(z-H)\right\}}{\alpha_{1} d^{2}} \log \left(\frac{y^{2}+\left[\alpha_{1}(2 n H+H+d)+\alpha_{2}(z-H)\right]^{2}}{y^{2}+\left[\alpha_{1}(2 n H+H-d)+\alpha_{2}(z-H)\right]^{2}}\right)+\frac{2 y}{d}\right\}\right]
\end{aligned}
$$

2.4.3 Linear slip

$$
\begin{aligned}
& u^{I}=\frac{b_{0}}{2 \pi \alpha_{1}}\left[\sum _ { n = 0 } ^ { \infty } T ^ { n } \left\{\alpha_{1}\left(1-\frac{2 n H+z}{d}\right)\left(\tan ^{-1} \frac{(2 n H+z-d) \alpha_{1}}{y}\right)\right.\right. \\
& -\alpha_{1}\left(1+\frac{2 n H+z}{d}\right)\left(\tan ^{-1} \frac{(2 n H+z+d) \alpha_{1}}{y}\right)+\frac{2 \alpha_{1}(2 n H+z)}{d}\left(\tan ^{-1} \frac{\alpha_{1}(2 n H+z)}{y}\right) \\
& \left.+\frac{y}{2 d} \log \left(\alpha_{1}^{2}(2 n H+z-d)^{2}+y^{2}\right)\left(\alpha_{1}^{2}(2 n H+z+d)^{2}+y^{2}\right)-\frac{y}{d} \log \left(\alpha_{1}^{2}(2 n H+z)^{2}+y^{2}\right)\right\} \\
& \quad+\sum_{n=1}^{\infty} T^{n}\left\{\alpha_{1}\left(1-\frac{2 n H-z}{d}\right)\left(\tan ^{-1} \frac{(2 n H-z-d) \alpha_{1}}{y}\right)\right. \\
& -\alpha_{1}\left(1+\frac{2 n H-z}{d}\right)\left(\tan ^{-1} \frac{(2 n H-z+d) \alpha_{1}}{y}\right)+\frac{2 \alpha_{1}(2 n H-z)}{d}\left(\tan ^{-1} \frac{\alpha_{1}(2 n H-z)}{y}\right) \\
& \left.\left.+\frac{y}{2 d} \log \left(\alpha_{1}^{2}(2 n H-z-d)^{2}+y^{2}\right)\left(\alpha_{1}^{2}(2 n H-z+d)^{2}+y^{2}\right)-\frac{y}{d} \log \left(\alpha_{1}^{2}(2 n H-z)^{2}+y^{2}\right)\right\}\right]
\end{aligned}
$$

for $0 \leq z<H$ and for $Z>H$ 


$$
\begin{aligned}
& u^{I I}=\frac{b_{0}}{2 \pi \alpha_{1}}\left[\sum _ { n = 0 } ^ { \infty } ( 1 + T ) T ^ { n } \left\{\alpha_{1}\left(1-\frac{\alpha_{1}(2 n H+H)+\alpha_{2}(z-H)}{\alpha_{1} d}\right)\right.\right. \\
& \times\left(\tan ^{-1} \frac{\alpha_{1}(2 n H+H-d)+\alpha_{2}(z-H)}{y}\right) \\
& -\alpha_{1}\left(1+\frac{\alpha_{1}(2 n H+H)+\alpha_{2}(z-H)}{\alpha_{1} d}\right)\left(\tan ^{-1} \frac{\alpha_{1}(2 n H+H+d)+\alpha_{2}(z-H)}{y}\right) \\
& +\frac{2\left[\alpha_{1}(2 n H+H)+\alpha_{2}(z-H)\right]}{d}\left(\tan ^{-1} \frac{\alpha_{1}(2 n H+z)+\alpha_{2}(z-H)}{y}\right) \\
& +\frac{y}{2 d} \log \left(y^{2}+\left[\alpha_{1}(2 n H+H+d)+\alpha_{2}(z-H)\right]^{2}\right)\left(y^{2}+\left[\alpha_{1}(2 n H+H-d)+\alpha_{2}(z-H)\right]^{2}\right) \\
& \left.\left.-\frac{y}{d} \log \left(y^{2}+\left[\alpha_{1}(2 n H+H)+\alpha_{2}(z-H)\right]^{2}\right)\right\}\right] \text {. }
\end{aligned}
$$

\subsubsection{Cubic slip}

The surface deformation due the cubic slip is:

$$
\begin{aligned}
& u^{I}=\frac{-b_{0}}{2 \alpha_{1}^{3} d^{3}}\left[( \sum _ { n = 0 } ^ { \infty } T ^ { n } + \sum _ { n = 1 } ^ { \infty } T ^ { n } ) \left\{-y\left(\frac{3}{2}\left(d^{2}-8 H^{2} n^{2}\right) \alpha_{1}^{2}+y^{2}\right)\right.\right. \\
& \left.\left. \pm \frac{1}{2}\left(\left(d^{2}-4 H^{2} n^{2}\right) \alpha_{1}^{2}+4 i n H y \alpha_{1}+y^{2}\right)^{3 / 2} \pm \frac{1}{2}\left(\left(d^{2}-4 H^{2} n^{2}\right) \alpha_{1}^{2}-4 i n H y \alpha_{1}+y^{2}\right)^{3 / 2}\right\}\right]
\end{aligned}
$$

where the upper sign ' + ' is for $y>0$ and the lower sign ' ' 'is for $y<0$.

for $0 \leq z<H$ and for $z>H$

$$
\begin{aligned}
& u^{I I}=\frac{-b_{0}}{2 \alpha_{1}^{3} d^{3}}\left[\sum _ { n = 0 } ^ { \infty } ( 1 + T ) T ^ { n } \left\{-y\left(\frac{3}{2} d^{2} \alpha_{1}^{2}-3 \alpha_{2}^{2}\left(\frac{\alpha_{1}}{\alpha_{2}}(2 n H+H)-H\right)^{2} 2+y^{2}\right)\right.\right. \\
& \pm \frac{1}{2}\left\{d^{2} \alpha_{1}^{2}-\alpha_{2}^{2}\left(\frac{\alpha_{1}}{\alpha_{2}}(2 n H+H)-H\right)^{2}+2 i \alpha_{2} y\left(\frac{\alpha_{1}}{\alpha_{2}}(2 n H+H)-H\right)^{2}+y^{2}\right\}^{3 / 2} \\
& \left.\left. \pm \frac{1}{2}\left\{d^{2} \alpha_{1}^{2}-\alpha_{2}^{2}\left(\frac{\alpha_{1}}{\alpha_{2}}(2 n H+H)-H\right)^{2}-2 i \alpha_{2} y\left(\frac{\alpha_{1}}{\alpha_{2}}(2 n H+H)-H\right)^{2}+y^{2}\right\}^{3 / 2}\right\}\right]
\end{aligned}
$$

\subsubsection{Elliptic slip}

The surface deformation due to an elliptic slip profile is:

$$
\begin{aligned}
& u^{I}=\frac{-b_{0}}{2 \alpha_{1} d}\left[( \sum _ { n = 0 } ^ { \infty } T ^ { n } + \sum _ { n = 1 } ^ { \infty } T ^ { n } ) \left(-y \pm \frac{1}{2} \sqrt{\left(d^{2}-4 H^{2} n^{2}\right) \alpha_{1}^{2}+4 i n H y \alpha_{1}+y^{2}}\right.\right. \\
& \left.\left. \pm \frac{1}{2} \sqrt{\left(d^{2}-4 H^{2} n^{2}\right) \alpha_{1}^{2}-4 i n H y \alpha_{1}+y^{2}}\right)\right]
\end{aligned}
$$


for $0 \leq z<H$ and for $z>H$

$$
\begin{aligned}
u^{I I}=\frac{-b_{0}}{2 \alpha_{1} d}[ & {\left[\sum_{n=0}^{\infty}(1+T) T^{n}(-y\right.} \\
\pm & \frac{1}{2} \sqrt{\left(d^{2}-(2 n H+H)^{2}\right) \alpha_{1}^{2}+2 H(2 n+1)\left(\alpha_{2} H+i y\right) \alpha_{1}-\left(\alpha_{2} H+i y\right)^{2}} \\
\pm & \left.\left.\frac{1}{2} \sqrt{\left(d^{2}-(2 n H+H)^{2}\right) \alpha_{1}^{2}+2 H(2 n+1)\left(\alpha_{2} H-i y\right) \alpha_{1}-\left(\alpha_{2} H-i y\right)^{2}}\right)\right]
\end{aligned}
$$

\section{Particular cases}

\subsection{Smooth-rigid contact}

When the interface $\mathrm{Z}=\mathrm{H}$ between the layer and the base is 'smooth-rigid', we have found that the expressions for the displacements and stresses of the elastic layer are the same as obtained from the equations (18)-(22) on taking $T=1$.

\subsection{Rough-rigid contact}

Similarly, we have also verified that the expressions for the displacements and stresses of the elastic layer corresponding to the 'rough-rigid' interface $z=H$ are the same as given by the equations (18)-(22) with $T=-1$.

\subsection{Uniform orthotropic elastic half-space}

On putting $\alpha_{1}=\alpha_{2}=\alpha, c_{1}=c_{2}=c$ and $T=0$, we get the deformation field due to strike-slip fault in a uniform orthotropic elastic half-space, which coincide with the results earlier obtained by Madan et al (2005).

\subsection{Isotropic elastic layered medium}

The results for the corresponding problem for an isotropic elastic layered medium can be obtained as a particular case of the above results on putting $\alpha_{1}=\alpha_{2}=1, c_{1}=\mu_{1}, c_{2}=\mu_{2}$ and $T=\left(\mu_{1}-\mu_{2}\right) /\left(\mu_{1}+\mu_{2}\right)$. Here $\mu_{1}$ and $\mu_{2}$ denote the rigidities of the isotropic elastic layer and the half-space, respectively.

\section{Numerical Results and Discussion}

In this section, we compare the displacements for perfectly bonded orthotropic elastic layered half-space with the corresponding displacements for homogenous orthotropic elastic half-space due to different slip profiles along a very long vertical strike-slip fault. Also, to examine the effect of interface ('perfect', 'smooth-rigid' and 'rough-rigid') between the orthotropic elastic layer and the base due to different slip profiles (uniform, parabolic and linear) along the vertical strike-slip faults, contour maps for displacements at different depth levels have been drawn.

We define, $Y=\frac{y}{H}, Z=\frac{Z}{H}, \gamma=\frac{d}{H}, U^{(1)}=\frac{u^{(1)}}{b_{0}}, U^{(2)}=\frac{u^{(2)}}{b_{0}}, \sigma_{12}^{(1)}=\frac{\tau_{12}^{(1)} H}{b_{0} c_{1}}$ and $\sigma_{13}^{(1)}=\frac{\tau_{13}^{(1)} H}{b_{0} c_{1}}$

etc. as dimensionless distances, displacements and stresses.

Baryte is the least soluble sulphate and the most abundant barium mineral in the Earth's crust. The main constituent of the mantle is believed to be magnesia silicate, mostly in the form of Olivine. The velocity of seismic waves through olivine (which is a major constituent of the mantle) is greater when parallel to the axis of the Olivine crystal than when perpendicular to the axis. Such dependence of seismic velocity on direction is called velocity anisotropy (i.e., the material is not perfectly isotropic). For numerical computations, we use the values of orthotropic elastic constants for Baryte material given by Love (1944) for the medium I. These constants are $\alpha_{1}=0.9824, \mathrm{c}_{1}=2.87 \times 10^{4} \mathrm{MPa}$. For medium II, we use the values of orthotropic elastic constants given by Verma (1960) for Olivine materials which are $\alpha_{2}=0.9894, c_{1}=8.10 \times 10^{4} \mathrm{MPa}$. 
. For all slip profiles considered, the slip decreases from a value $b_{0}$ at the surface to zero at the depth ' $d$ '. If the surface slip $b_{0}$ and the fault depth ' $d$ ' are assumed to be the same for all cases, then assuming the source potency as $\int_{0}^{d} b(h) d h$ per unit length of the fault, is different for different slip profiles. This yield

$$
d_{1}=\frac{2}{3} d_{2}=\frac{1}{2} d_{3}=\frac{3 \pi}{16} d_{4}=\frac{\pi}{4} d_{5}=d \text { (say) }
$$

where $d_{1}$ is the fault depth for the uniform slip model and $d_{2}, d_{3}, d_{4}$ and $d_{5}$ are, respectively, the fault depths for the parabolic, linear, cubic and elliptic profiles.

To compare the deformation due to non-uniform slip profiles with the corresponding deformation due to uniform slip in the same elastic medium, the source potency should be the same and it can be achieved by varying the fault depth ' $d$ ', keeping the surfaceslip constant. In the present study, we have compared the results of an orthotropic elastic layered medium with an orthotropic elastic half-space for different slip profiles with their respective source potencies.

Comparison between the displacements, due to different slip profiles, along a very long vertical strike-slip fault in orthotropic elastic layered medium and uniform orthotropic elastic half-space also exhibits the effect of different slip profiles and of fault depths. For this, we consider $\gamma=1(d=H)$ and $\gamma=\frac{2}{3}\left(d=\frac{2}{3} H\right)$, i.e. when the source extends upto the depth of the layer and completely lies in the layer. In figures 2(a)-2(e), the horizontal dimensionless displacements parallel to the fault at the surface $Z=0$ and $\gamma=1$ for all slip profiles have been shown and at the sub-surface $Z=\frac{1}{2}, \frac{2}{3}$ and $\gamma=\frac{2}{3}$, the dimensionless displacement for uniform, parabolic and linear slips are shown in figures 3(a)-4(c). From these figures, it is found that in case of uniform, parabolic and linear slips, for fixed value of ' $\gamma$ ', as the values of $Z$ increases, the difference increases. It is also observed

that $U^{(1)}$ is anti-symmetric with respect to the distance from the fault $Y$ which is, in fact, the property of the problem considered.

The contour maps in figures 5(a)-7(c) show the displacement field around a long vertical strike-slip fault for a uniform, parabolic and linear slip profiles for different interfacing ('perfect', 'smooth-rigid' and 'rough-rigid') between orthotropic elastic layer and the orthotropic half-space. The displacement is measured in units of $10^{-3}$. Contours in figures 5(a)-5(c) show the subsurface displacement for uniform slip for $\gamma=\frac{2}{3}$ and for parabolic and linear slips, the displacement field for $\gamma=1$ are shown in the figure 6(a)-7(c). For uniform, parabolic and linear slip profiles, these contours also show that the displacement field significantly influenced by the nature of interfacing ('perfect', 'smooth-rigid' and 'rough-rigid') between orthotropic elastic layer and orthotropic elastic half-space. It is found from these figures that the displacement parallel to the strike of the fault at the lower edge of the fault is discontinuous for uniform slip. However, this discontinuity is not present in the displacement field for parabolic and linear slip profiles. It is due to the shape of the slip model considered. For uniform slip, discontinuity is constant and for nonuniform slip discontinuity becomes zero at the lower edge of the fault, i.e., where the fault is locked. 

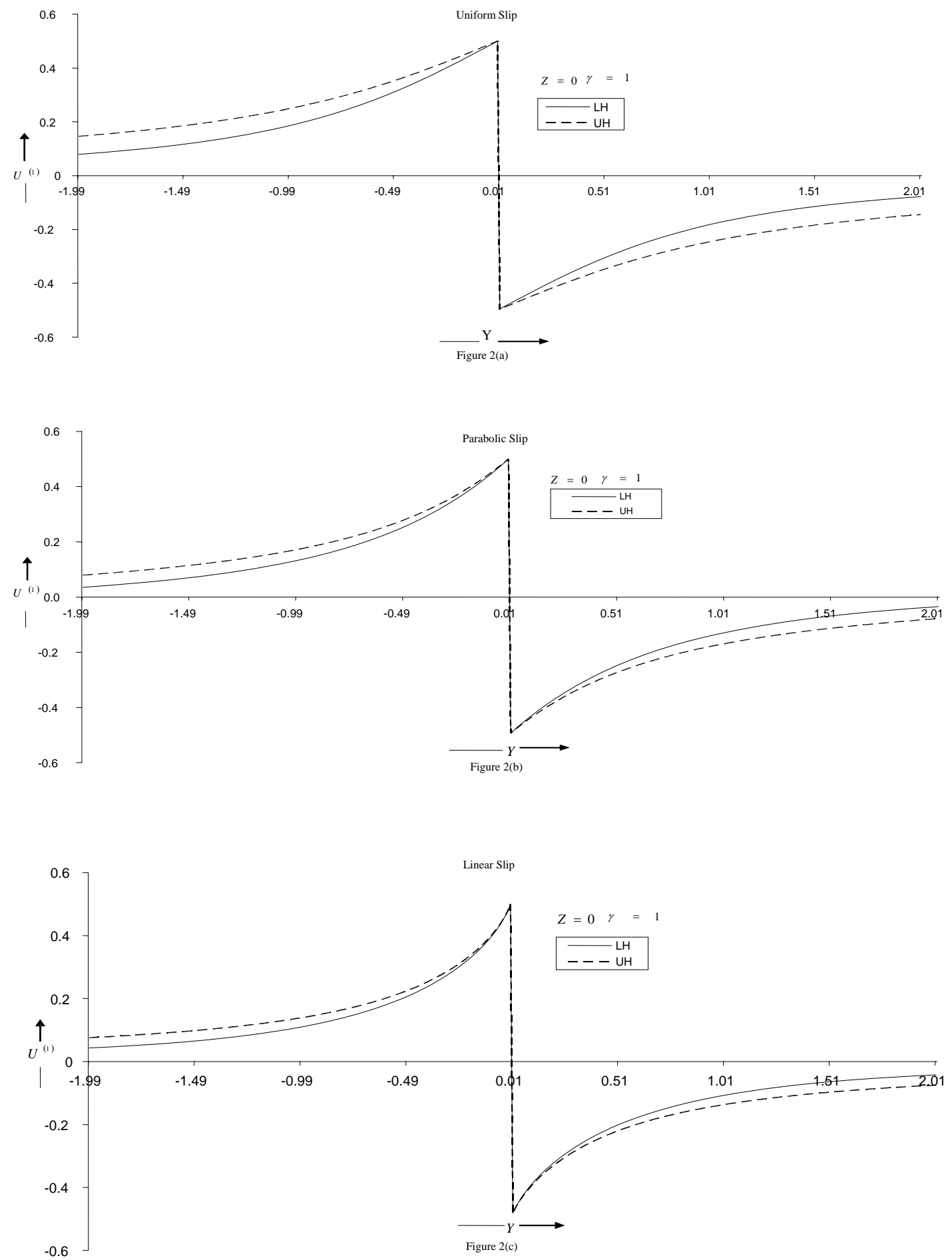

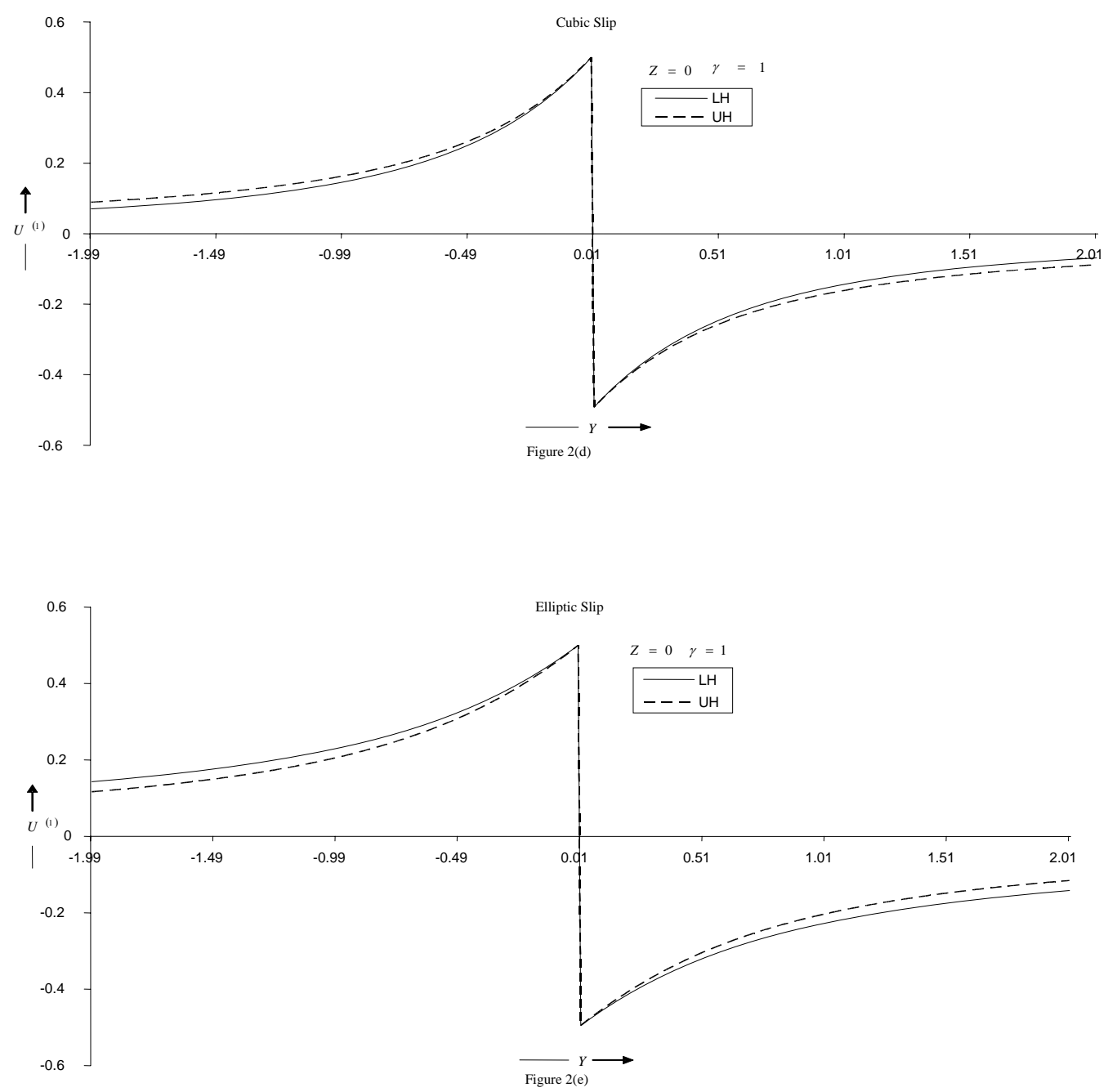

Figure 2. Variation of the dimensionless displacement $U^{(1)}$ of orthotropic elastic layered half-space (LH) with the corresponding dimensionless displacement for a uniform orthotropic elastic half-space (UH) from the upper edge of the fault $Y$ for $Z=0$ and $\gamma=1$ for (a) uniform slip (b) parabolic slip (c) linear slip (d) cubic slip (e) elliptic slip.

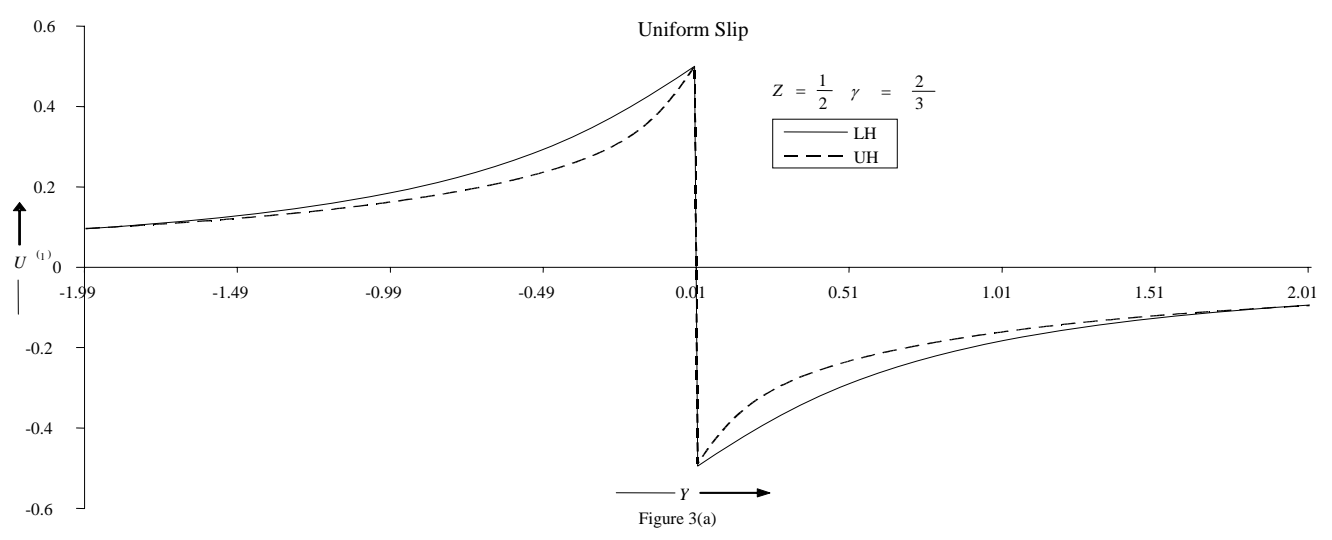



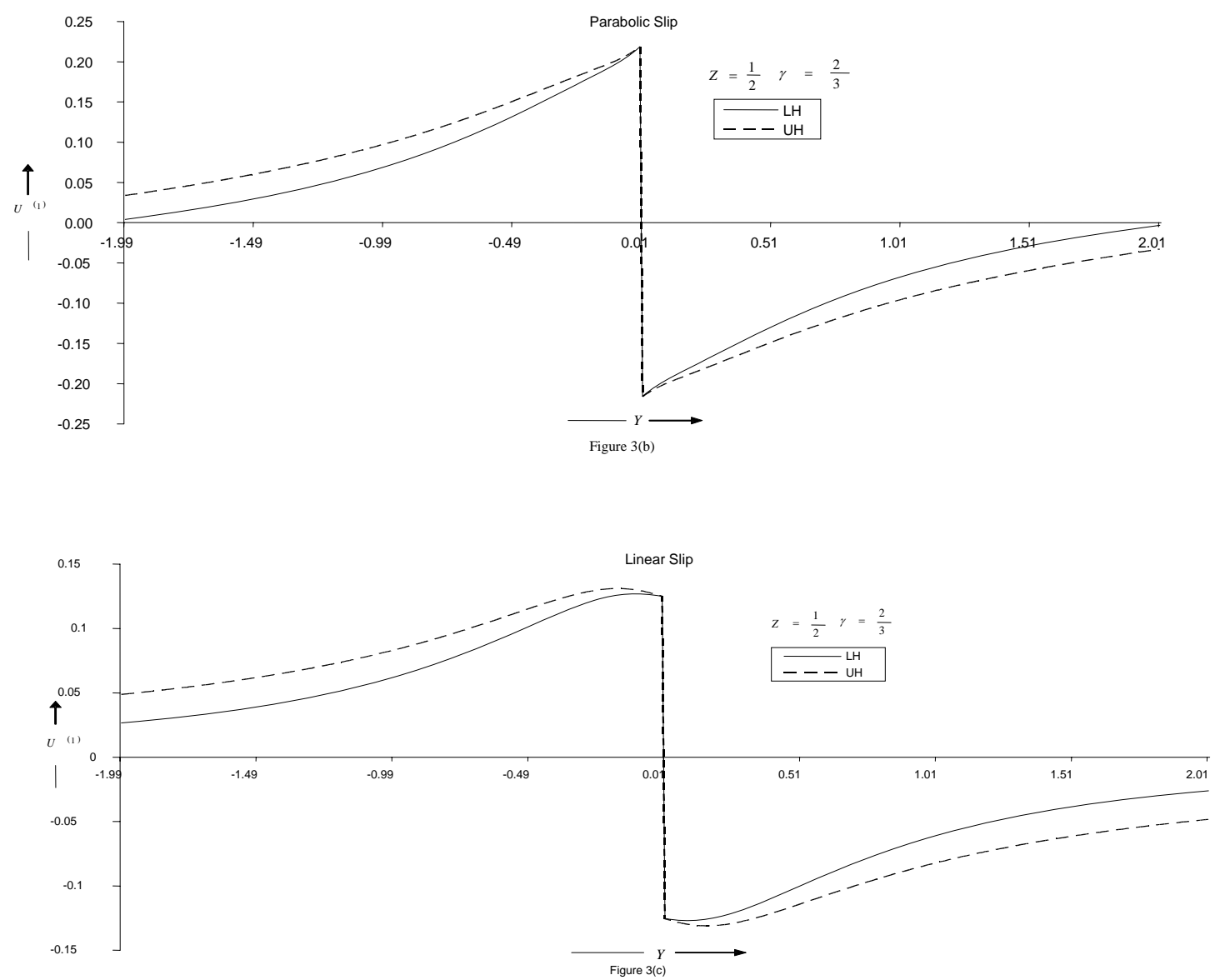

Figure 3. Variation of $U^{(1)}$ with $Y$ for $Z=\frac{1}{2}$ and $\gamma=\frac{2}{3}$ for (a) uniform slip (b) parabolic slip (c) linear slip. Notations as in figure 2. 

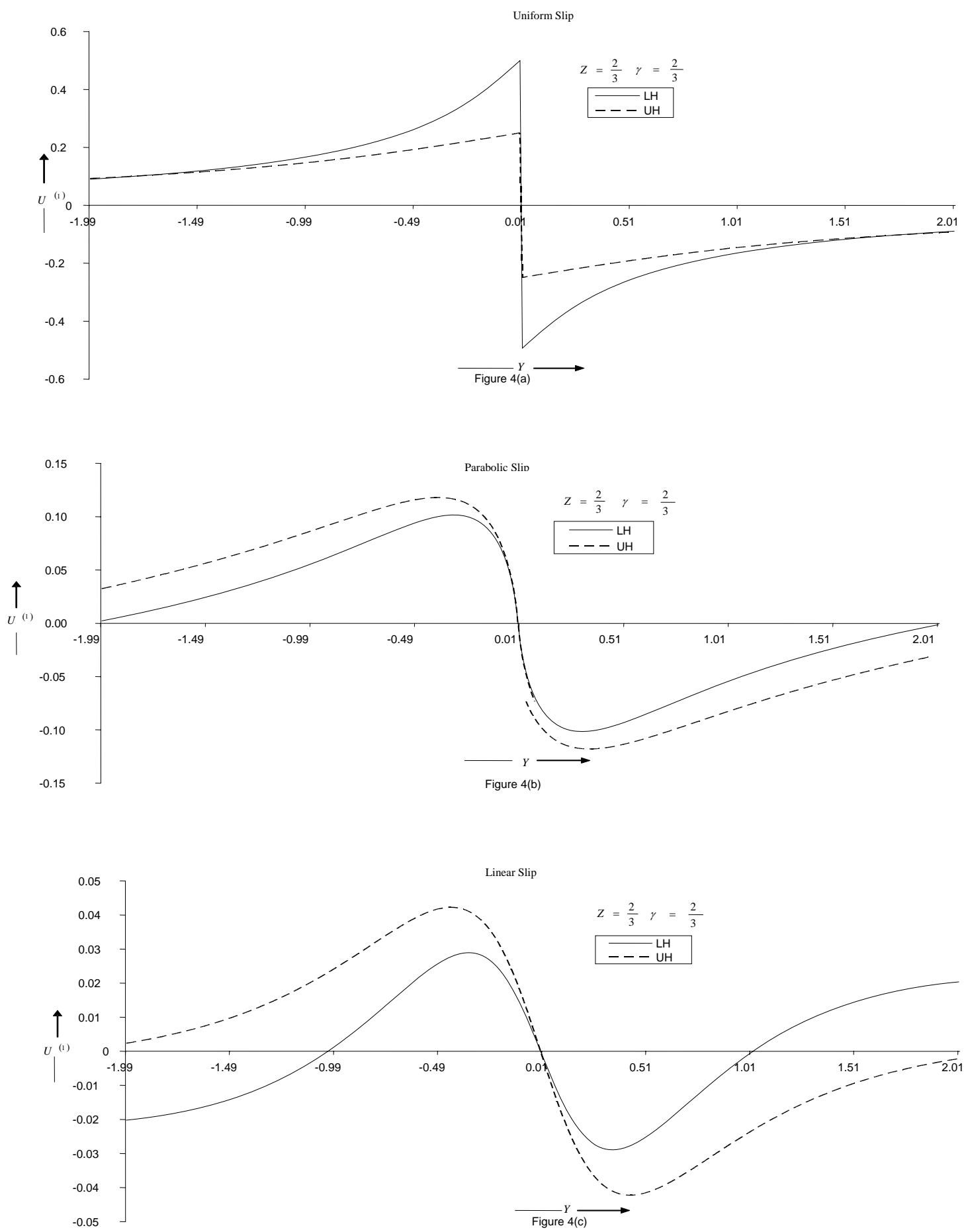

Figure 4. Variation of $U^{(1)}$ with $Y$ for $Z=\frac{2}{3}$ and $\gamma=\frac{2}{3}$ or (a) uniform slip (b) parabolic slip (c) linear slip. Notations as in figure 2. 

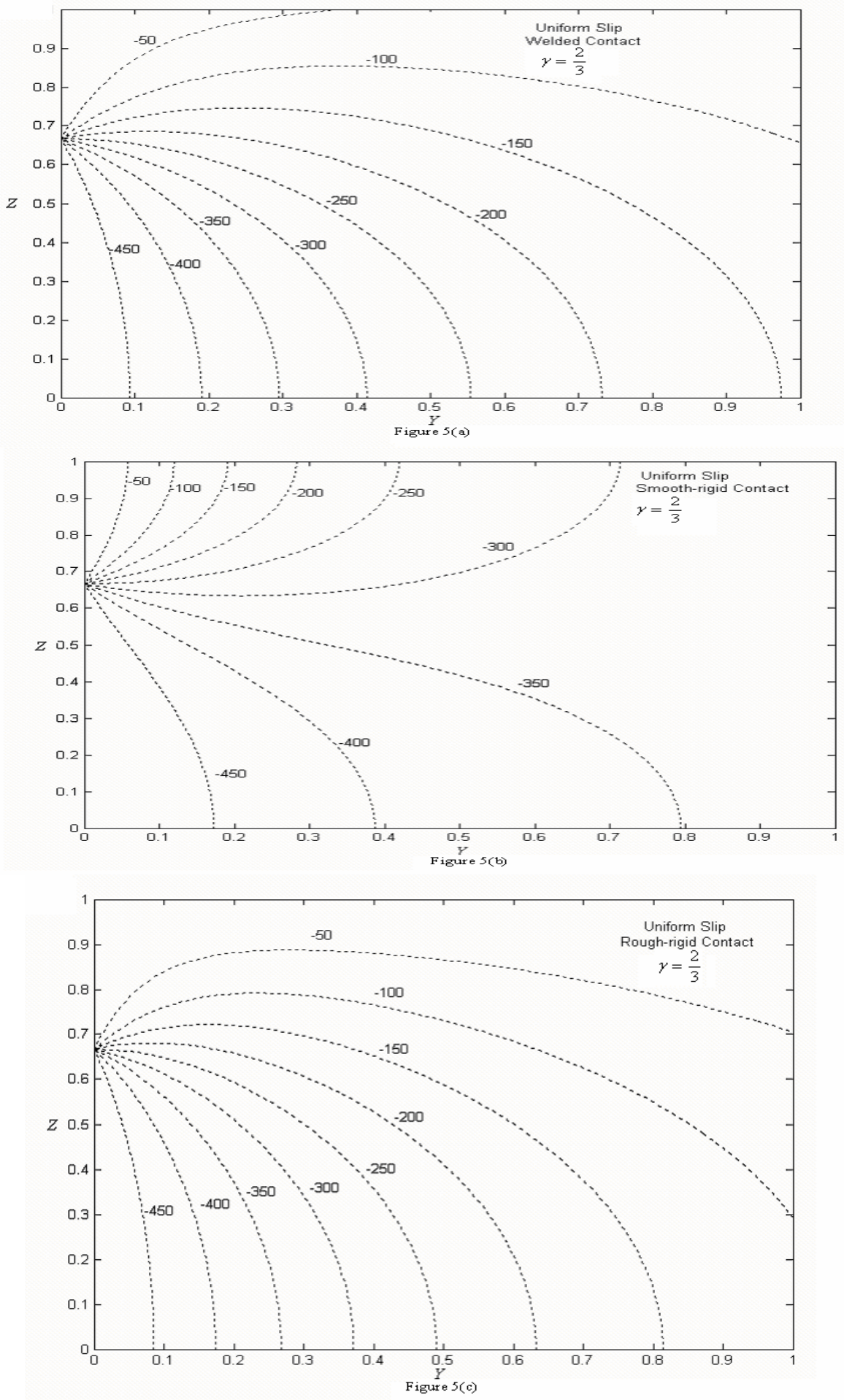

Figure 5. Contour map for the displacement $U^{(1)}$ parallel to strike of a vertical strike-slip fault for uniform slip for $\gamma=\frac{2}{3}$ due to (a) welded contact (b) smooth-rigid contact (c) rough-rigid contact. The distance is measured in units of $10^{-3}$. 

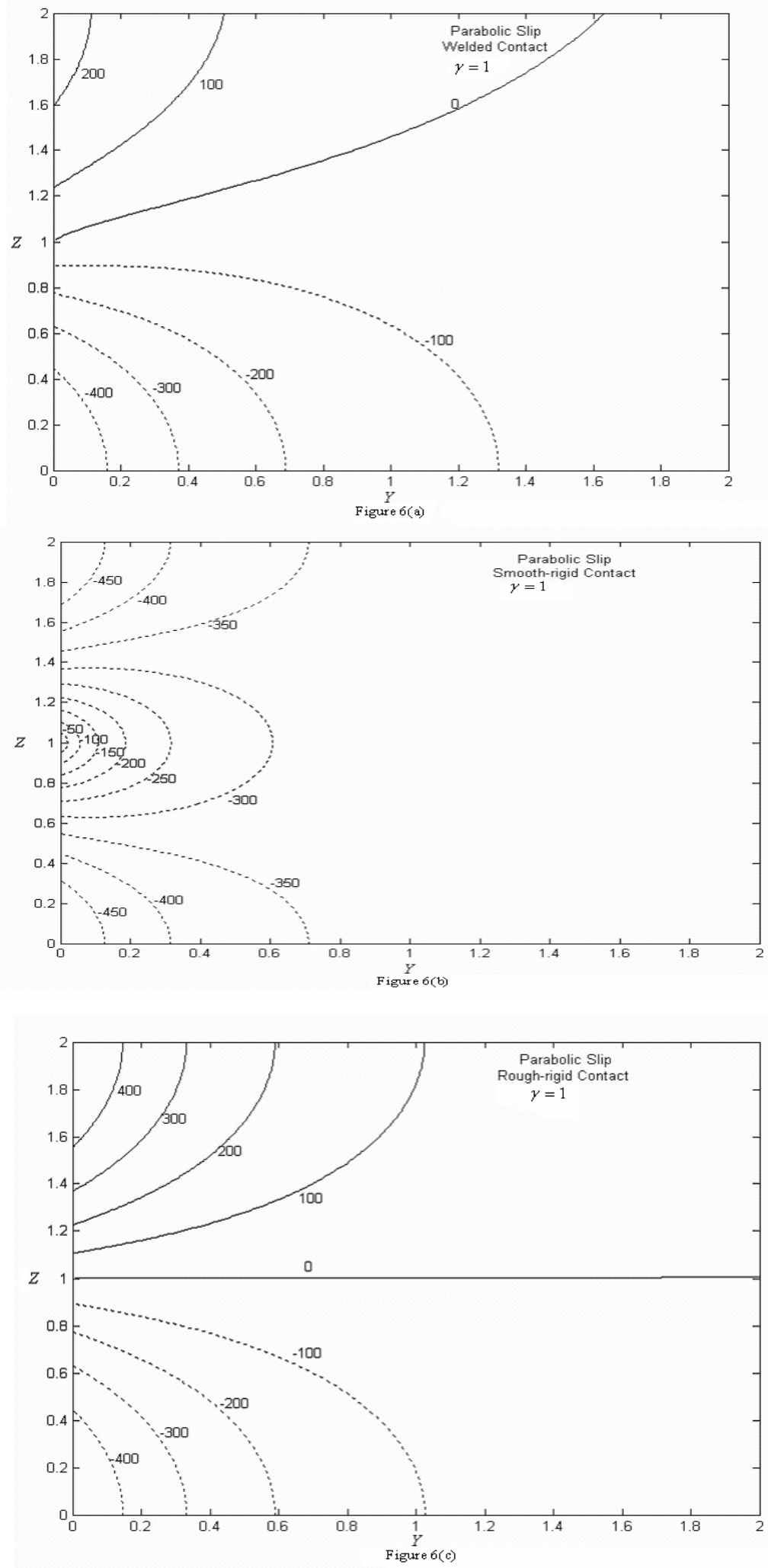

Figure 6. Contour map for the displacement $U^{(1)}$ for parabolic slip for $\gamma=1$ due to (a) welded contact (b) smoothrigid contact (c) rough-rigid contact. 

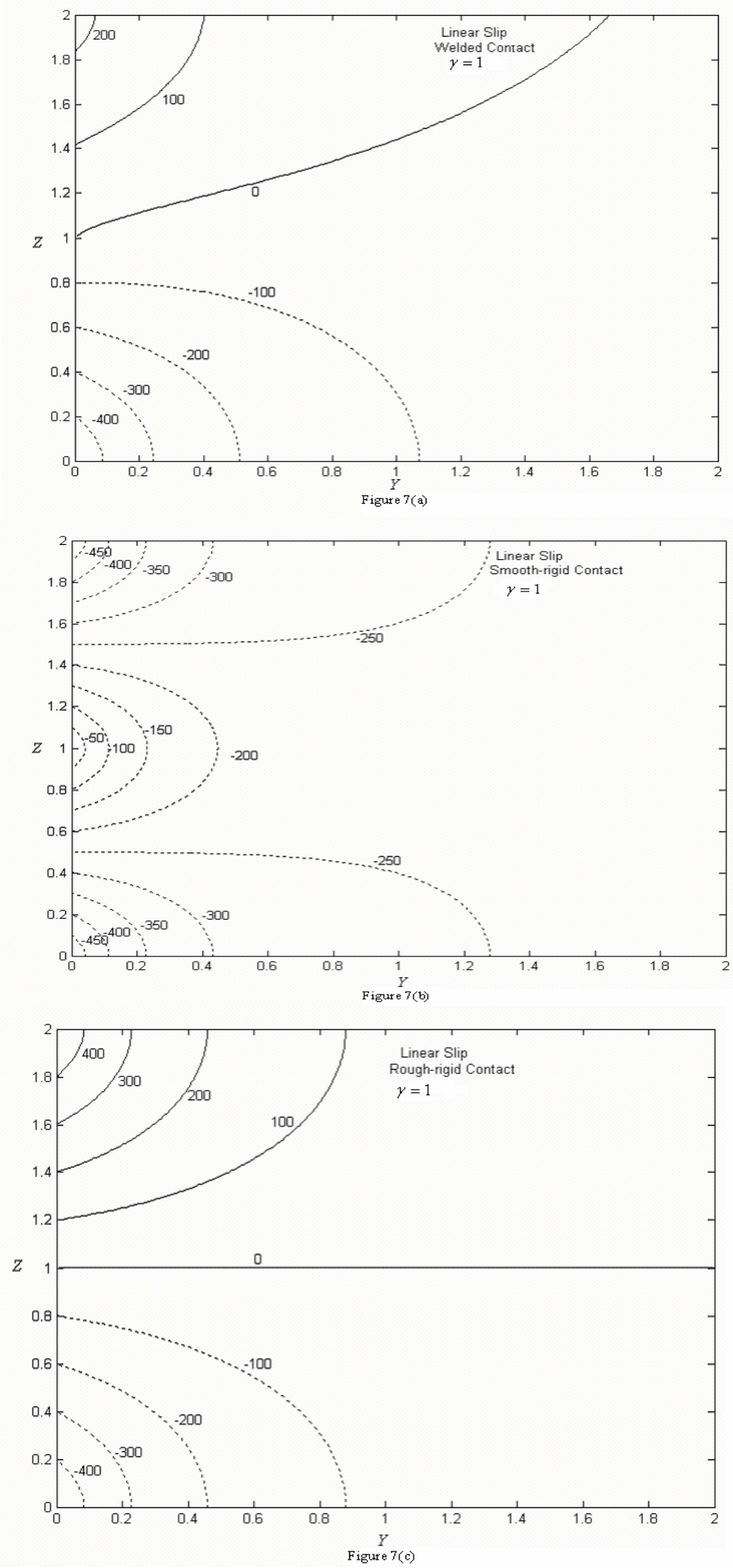

Figure7. Contour map for the displacement $U^{(1)}$ for linear slip for $\gamma=1$ due to (a) welded contact (b) smooth-rigid contact (c) rough-rigid contact.

Static dislocation models are mainly applied to analyze the residual deformation of a medium caused by earthquake faults. The analysis of source parameters of tsunamigenic earthquakes indicates that non-uniform distribution along the fault has significant 
effect on co-seismic displacement field (Satake, 1994; Piatanesi, 1996 and Geist, 2005). In engineering, elastic layer represents an elastic plate while in Geophysics, it represents a lithosphere. For shallow earthquakes, the elastic layer of our problem may be identified with topmost brittle region of the crust and the perfectly bonded elastic base with the crustal zone. For intermediate earthquakes, the model of Earth consisting of lithosphere lying over an asthenosphere may be used. It has been established that in a Palaeozoic sedimentary rocks in Enola, Arkansas, USA, earthquake source lies (Crampin, 1994). Such sedimentary rocks may be represented in our model by layer and the bottom of the layer may be taken as rough-rigid.The smooth-rigid interface condition is applicable to engineering problems where there is a possibility of the layer (oil reservoir rocks) slipping over base and may also be used to study the effect of an internal horizontal boundary which is lubricated and the shear stress components and vertical components of the displacement vector become zero there. The present problem highlights the anisotropy of the elastic structure in sub-lithospheric oceanic mantle detected by seismic methods. This study could also be used to predict near-field displacements useful for the inverse source studies from the observed static displacement following shallow large earthquakes.

\section{Acknowledgement}

We are thankful to the reviewers for the improvement in the paper.

\section{References}

Crampin S. 1994. The Fracture Criticality of Crustal Rocks. Geophysical Journal International. Vol. 118, No.2, pp. 428-438.

Eshelby J. D. 1957. The determination of the elastic field of an ellipsoidal inclusion and related problems. Proceedings of Royal Society A and Series B. Vol. 241, No. 1226, pp. 376-396.

Burridge R, Knopoff L.1964. Body forces equivalents for seismic dislocations. Bulletin of the Seismological Society of America, Vol. 54, No. 6A, pp. 1875-1888.

Garg N.R, Sharma R.K.1992. Deformation of an elastic layer coupling in different ways to a base due to a very long vertical strike-slip dislocation. Journal of Earth System Science. Vol. 101, No. 3, pp.255-268.

Garg N.R, Madan D.K, Sharma R.K.1996. Two-dimensional deformation of an orthotropic elastic medium due the seismic sources. Physics of the Earth and Planetary Interiors. Vol. 94, No.1, pp. 43-62.

Geist E.L.2005. Local Tsunami Hazards in the Pacific Northwest from Cascadia Subduction Zone Earthquakes. USGS Professional Paper. Vol. 1661 (B), pp.1-17.

Kasahara K.1964. A strike-slip fault buried in a layered medium. Bulletin of the Earthquake Research Institute, Vol .42, pp. 609619.

Love A.E.H.1944. A Treatise on the Mathematical Theory of Elasticity. Dover Publications: New York.

Madan D.K, Singh K, Aggarwal R, Gupta A. 2005. Displacements and stresses in anisotropic medium due to non-uniform slip along a very long strike-slip fault. ISET Journal of Earthquake Technology, Vol. 42, No.1, pp.1-11.

Maruyama T. 1966. On two dimensional elastic dislocations in an infinite and semi-infinite medium. Bulletin of the Earthquake Research Institute. Vol. 44, No. 3, pp. 811-87.

Piatanesi A, Tinti S, Gavagni I. 1996.The slip distribution of the 1992 Nicaragua earthquake from tsunami run-up data: Geophysical Research Letters, Vol. 23, No.5, pp.37-40.

Ruina A, Katzman Y, Conrad G, Horowitz F.G. Some theory and experiments related to frictional behavior of rocks at low normal stress. Pure and Applied Geophysics. 1-82. http://ruina.tam.cornell.edu/research/topics/friction_and_fracture/Ruina_Pageoph_\%201986.pdf

Satake K.1994. Mechanism of the 1992 Nicaragua tsunami earthquake. Geophysical Research Letter, Vol. 21, No. 23, pp. 25192522.

Singh S.J, Punia M and Rani S. 1994. Crustal deformation due to non-uniform slip along a long fault. Geophysical Journal International. Vol. 118, No. 2, pp. 411-427.

Starr A.T. 1928.Slip in a crystal and rupture in a solid due to shear. Proceedings of Cambridge Philosophical Society. Vol. 24, pp. 489-500.

Sokolnikoff I.S.1956. Mathematical Theory of Elasticity. McGraw-Hill: New York.

Verma R.K.1990. Elasticity of some high-density crystals. Journal of Geophysical Research, Vol.65, pp.757-766.

Wang R, Wu H.L.1983. Displacement and stress fields due to a non-uniform slip along a strike-slip fault. Pure and Applied Geophysics.; Vol.121, No. 4, pp.601-609.

Yang M, Toks $\ddot{\text { z }}$, M. N. 1981.Time dependent deformation and stress relation after strike-slip earthquakes. Journal of Geophysical Research. Vol. 86, No. B4, pp. 2889-2901.

Biographical notes

Shamta Chugh received M.Sc and M.Phil from M.D.University Rohtak India in 1999 and 2000, respectively. She is research scholar in Department of Mathematics, Guru Jambheshwar University of Science and Technology, Hisar, India. 
Dinesh Kumar Madan received M.Sc. and Ph.D from M.D.University Rohtak ,India in 1990 and 1998, respectively. He is Associate Professor in Mathematics and Head of the Department of Applied Sciences \& Humanities, The Technological Institute of Textile and Sciences Bhiwani, India..He is a Life Member of Indian Mathematical Society. He has written a book on Engineering Mathematics-I.

Kuldip Singh is a Professor in the Department of Mathematics, Guru Jambheshwar University of Science \& Technology Hisar, India. He has more than 20 years experience in teaching and research. He has also presented number of research articles in National and International conferences. He has also written few books in Mathematics.

Received January 2011

Accepted February 2011

Final acceptance in revised form March 2011 